(C) 2010 IEEE. Reprinted, with permission, from Nogués, 0., Ribó, S., Arco, J. C., Cardellach, E., Rius, A., València, E., A. Camps, van der Marel, H., Martín-Neira, M., The proof of concept for 3-cm altimetry using the PARIS interferometric technique, Proceedings of IEEE International Geoscience and Remote Sensing Symposium (IEEE IGARSS), and july/2010.

This material is posted here with permission of the IEEE. Such permission of the IEEE does not in any way imply IEEE endorsement of any of CSIC products or services. Internal or personal use of this material is permitted. However, permission to reprint/republish this material for advertising or promotional purposes or for creating new collective works for resale or redistribution must be obtained from the IEEE by writing to pubs-permissions@ieee.org.

By choosing to view this document, you agree to all provisions of the copyright laws protecting it. 


\title{
THE PROOF OF CONCEPT FOR 3-CM ALTIMETRY USING THE PARIS INTERFEROMETRIC TECHNIQUE
}

\author{
O. Nogués-Correig ${ }^{1}$, S. Ribó ${ }^{1}$, J.C. Arco ${ }^{1}$, E. Cardellach ${ }^{1}$, A. Rius ${ }^{1}$, E. Valencia ${ }^{2}$, J.M. Tarongí ${ }^{2}$, A. Camps ${ }^{2}$, \\ H. van der Marel ${ }^{3}$, M. Martín-Neira ${ }^{4}$ \\ ${ }^{1}$ ICE-IEEC/CSIC, Barcelona, Spain \\ ${ }^{2}$ Universitat Politècnica de Catatlunya, IEEC-CRAE/UPC, Barcelona, Spain \\ ${ }^{3}$ TUD, Delft, The Netherlands \\ ${ }^{4}$ ESTEC/ESA, Noordwijk, The Netherlands
}

\begin{abstract}
Index Terms- GNSS, GNSS Remote Sensing, GNSS-
\end{abstract} Reflections, PARIS, Altimetry

\section{INTRODUCTION}

Since its suggestion in 1993, the altimetric and scatterometric capabilities of the PAssive Reflectometry and Interferometry System (PARIS) [1] have been tested extensively from ground- air- and even space-based experiments [2, e.g.]. The concept is based on the use of the Global Navigation Satellite System (GNSS) signals reflected off the Earth (Ocean, Ice, Land), in a bi-static radar configuration. ESA has proposed to use the PARIS Interferometric Technique at the future PARIS In-Orbit Demonstrator Instrument (PARIS-IOD) [3]. This is a novel instrumental approach with respect to previous PARIS instruments, which obtained the observables through crosscorrelating direct and reflected signals against a GPS signal model (or replica). The limit of such an altimetric approach was given by the availability of the GNSS codes. The new interferometric technique aligns the direct and reflected signals both in delay and Doppler and computes directly crosscorrelation between the received signals (not against replicas of their codes). The principal advantage of this new technique is that in addition to public GPS signals, also encrypted signals can be used simultaneously, increasing the available signal bandwidth and power, and thus the expected precision of the altimetric measurement.

This project contributes to the PARIS-IOD concept trying to demonstrate the suitability of the interferometric technique for altimetric purposes, as well as to study and demonstrate the proposed calibration techniques for the PARIS-IOD instrument. We aim to confirm whether the interferometric technique is capable of measuring GPS signal delay differences with an associated 1 sigma error of $3 \mathrm{~cm}$ with 1 second

Project under ESA RFQ/3-127-47/09/NL/JD grant and Spanish AYA2008-05906-C02-02/ESP Project.
Table 1. Characteristics of the PIR Receiver.

\begin{tabular}{ll}
\hline RF Bandwidth: & programmable, $8 \mathrm{MHz}$ to $80 \mathrm{MHz}$, \\
& in $580 \mathrm{kHz}$ steps \\
Sampling rate: & $80 \mathrm{HMHz}$ \\
\# cplx. correlators: & $320 \mathrm{lags}$ \\
Inter-lag delay: & $12.5 \mathrm{~ns}(3.75 \mathrm{~m})$ \\
Coh. Int. Time: & $1 \mathrm{~ms}$ \\
\hline
\end{tabular}

of observation time.

The first step to accomplish the objective of the activity is to build the appropriate receiver, able to perform the direct correlation between the two signals. This has been achieved by modifying the existing dedicated full-custom GNSS-reflection receiver GPS Open Loop Differential RealTime Receiver (GOLD-RTR) [4], now called PARIS Interferometric Receiver (PIR). Although the instrument proposed for the PARIS-IOD will have beam-forming antennas, our proof-of-concept instrument will operate with real aperture antennas as we will concentrate our effort on developing the real-time digital signal processing algorithms. The details of the PIR are given in Table 1.

\section{SPIRENT SIMULATOR EXPERIMENT}

The experiment setup designed to determine the capabilities of the PARIS Interferometric Technique is shown in Figure 1. The GPS signals are simulated using a GPS simulator equipment. The simulated signals include all visible GPS signals for a given simulation time, instrument location and dynamics, and one reflected signal, for only one PRN, on a perfectly reflecting flat surface [5]. These signals are fed into a power splitter and divided into three paths. The first one is injected into link 1 of the PIR. This first signal is named $N$ (Navigation). Link 1 in the PIR is used to obtain standard GPS observables (pseudo-ranges, Doppler, position, time, ...) using an embedded commercial GPS receiver. The other two GPS signals at the output of the power splitter correspond to the 


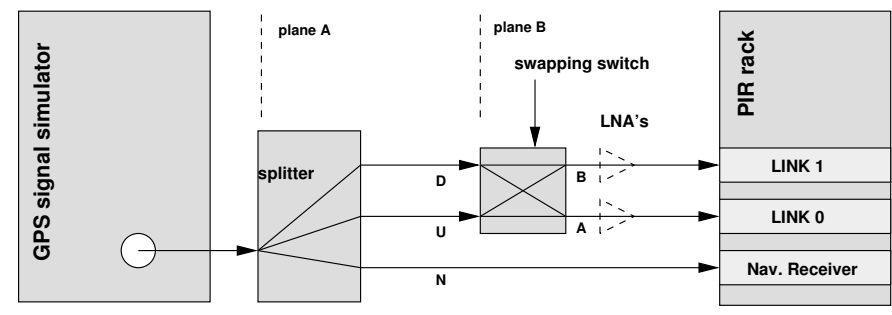

Fig. 1. Proposed experiment set-up using a GPS simulator environment.

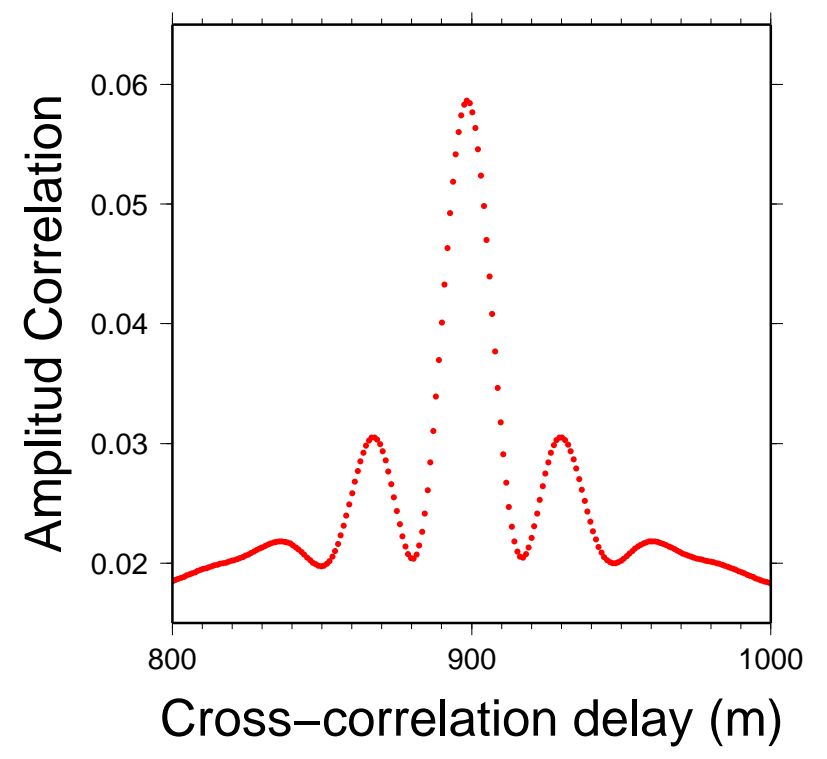

Fig. 2. Cross-correlation between direct and reflected signals ( $\mathrm{L} 1 \mathrm{C} / \mathrm{A}+\mathrm{P}+\mathrm{M}$ codes) generated by the SPIRENT (1:4 interpolation factor).

signals acquired by the up $(U)$ and down $(D)$ looking antenna arrays of PARIS-IOD. Using the switch, the $U$ and $D$ signals can be fed into links 0 or 1 using the swapping technique proposed in [6]. Link 0 would correspond to the front-end $A$ and link 1 to front-end $B$ in [6]. The PARIS interferometric technique is implemented by cross-correlating the signals of links 0 and 1 . LNAs are placed between the switch and the GOLD-RTR to simulate the presence of an LNA in the real PARIS-IOD.

\subsection{Group Delay Preliminary Results}

General speaking, only group-delay can be measured from GPS signals scattered off the Ocean surface. Therefore, the results presented are based in group-delay measurements solely (no carrier-phase delays).

The SPIRENT simulation tests were conducted on June 22, 2010. A large sequence of experiments was performed, in which different combinations of the delay lines, swapping, and calibration parameters were tested. The preliminary re-
Table 2. SPIRENT results for experiment \#14 after 33samples averaging (see Figure 4). Delays in centimeter with respect to first averaged-observation. $\rho_{n}$ and $\rho_{m}$ for SPIRENT-nominal and PIR measured delays respectively. $\sigma_{1 \text { sec }}$ corresponds to the actual dispersion of the data.

\begin{tabular}{c|r|rrr}
\hline $\begin{array}{c}\mathbf{T} \\
(\mathbf{s o d})\end{array}$ & $\begin{array}{r}\rho_{\mathbf{n}} \\
(\mathbf{c m})\end{array}$ & $\begin{array}{r}\rho_{\mathbf{m}} \\
(\mathbf{c m})\end{array}$ & $\begin{array}{r}\sigma_{\mathbf{1 s e c}} \\
(\mathbf{c m})\end{array}$ & $\begin{array}{r}\left|\rho_{\mathbf{n}}-\rho_{\mathbf{m}}\right| \\
(\mathbf{c m})\end{array}$ \\
\hline $300-359$ & 0 & ref & 1.8 & ref \\
$360-419$ & 1 & 0.8 & 1.8 & 0.2 \\
$420-479$ & 2 & 1.8 & 2.0 & 0.2 \\
$480-539$ & 5 & 4.6 & 1.8 & 0.4 \\
$540-599$ & 10 & 9.5 & 2.0 & 0.5 \\
$600-659$ & 20 & 19.5 & 2.0 & 0.5 \\
$660-719$ & 50 & 49.7 & 2.1 & 0.3 \\
$720-779$ & 100 & 100.0 & 2.1 & 0.0 \\
\hline
\end{tabular}

sults presented in this paper focus on two experiments solely, and only use observations taken in standard mode (i.e. no calibration measurements, no swapping of the channels). Those represent 33 seconds in each minute of measurements, which are not always sequential because the instrument loops around a sequence of calibration-test modes. The simulations were based on the GNSS status corresponding to March 62010. The power of the direct and reflected signals were in general selected to mimic the expected signal-to-noise ratio in the Bridge Experiment, that has been estimated as $40.4 \mathrm{~dB}$ at the correlation peak (see Section 3 and Figure 3 for the used formula). An example of the obtained cross-correlation waveforms is presented in Figure 2. As shown in e.g. [4], the approach used so far, which usually implies the correlation of the signals with the replica of the known, C/A, code, would have resulted in a \pm 300 meter wide triangle. Instead, the full correlation of both signals contains other GPS codes, of wider bandwidth ( $\mathrm{P}$ and $\mathrm{M})$, which introduce narrower main and secondary peaks.

Experiment \#14 consisted of a sequence of minutes of reflected data, increasing stepwise the delay of the reflected signal by re-programming of the SPIRENT simulator. Figure 3 provides more details on this experiment. The SPIRENT was programmed to introduce delay-jumps of 1 centimeter to 1 meter, in logarithmic steps (see 2 nd column in Table 2 or details in Figure 3). The formal uncertainty of the retrieved peak-locations of 1-second averaged waveforms varies between 1.3 to $2.8 \mathrm{~cm}$. The actual 1 -second dispersion, as shown in Table 2, corresponds to 1.8 to $2.1 \mathrm{~cm}$, all better than $3-\mathrm{cm}$. Figure 4 presents the measured delays. All the jumps introduced by the SPIRENT are detectable, even at 1-cm level. As shown in Table 2, the difference between the incremental delay measured by the PIR and the one actually programmed in the SPIRENT has a maximal value of $0.5 \mathrm{~cm}$.

Although the configured SNR should relate to the Bridge 


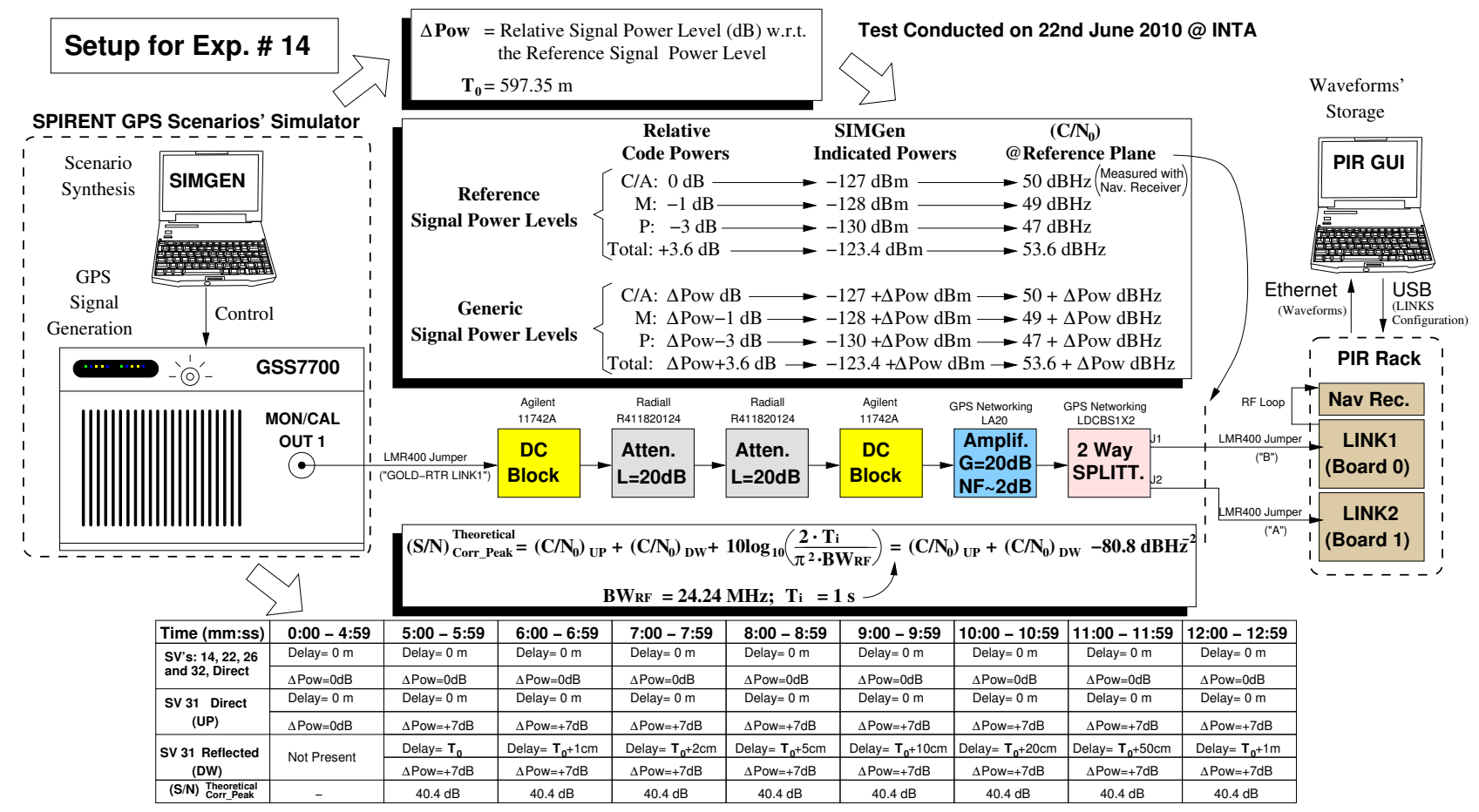

Fig. 3. Summarized set-up for experiment \#14.

Experiment, it is likely that some effects will degrade the performance in other scenarios (higher altitudes of the receiver, rougher sea surface conditions, instrumental, etc). In order to inspect the effect of the signal-to-noise degradation into the altimetric performance, Experiment \#17 gradually swept a range of $32 \mathrm{~dB}$ in the signal-to-noise ratio, around the nominal $40.4 \mathrm{~dB}$. The resulting 1 -second dispersion depends on the carrier signal-to-noise ratio as shown in Figure 5, from where we can deduce that $\sim 3 \mathrm{~dB}$ degradation with respect to the expected Bridge Experiment one ( $40.4 \mathrm{~dB})$ might still produce 1 sigma error below $3-\mathrm{cm}$ with 1 second observations. This degradation could be introduced by non-ideal conditions.

\section{BRIDGE EXPERIMENT}

The Bridge Experiment is planned for the second week of July 2010. The set-up for the bridge experiment is shown in Figure 6. This experiment requires independent calibration data to be provided on the sea-level height with respect to the main GNSS-R antennae; thus, a number of elements need to be surveyed accurately. The elements are $(1)$ The $\approx 1 \mathrm{~m}$ diameter uplooking antenna $(\mathbf{U})$ pointing to a fixed direction of the sky, where the selected GPS satellites will transit. The pointing direction of this antenna can be adjusted with a mechanical tilt (20 degrees respect to Zenith/Nadir), but it will not perform any tracking during the experiment. (2) An upward looking hemispherical antenna (N) which is used to obtain navigation observables. The position of this antenna is retrieved using a

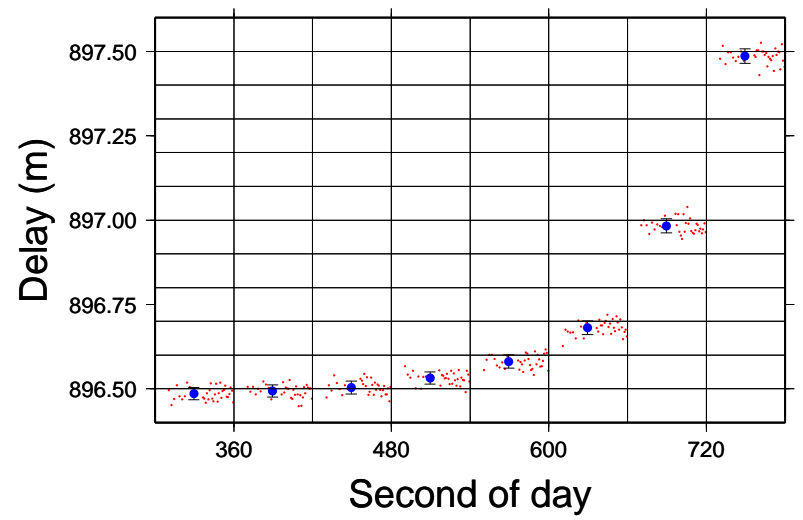

Fig. 4. Group-delays measured with the PIR during experiment \#14, 33 samples per minute, at 1 second integration each (red) and the 33-samples means (blue). 


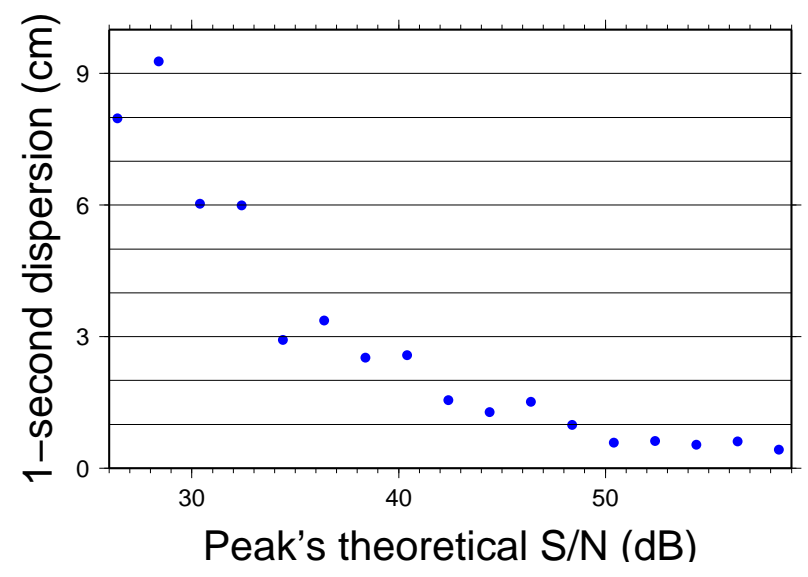

Fig. 5. 1-second dispersion of the PIR measured delays as function of the carrier signal-to-noise ratio in experiment \#17 (assuming $T_{i}=1 \mathrm{~s}, B W_{R F}=24.24 \mathrm{MHz}$ ). $40.4 \mathrm{~dB}$ is the expected SNR in the Bridge Experiment conditions.

GPS receiver. The signal of antenna $\mathbf{N}$ is also injected into the NAV inputs of the GOLD-RTR and PIR racks. (3) $\mathrm{A} \approx 1 \mathrm{~m}$ diameter down-looking antenna $\mathbf{D}$ that points to a fixed direction towards the sea, where the GPS reflected signals will transit as well.

The PARIS interferometric antennas are antennas $\mathbf{U}$ and D. It is intended to perform traditional model crosscorrelations (U, D) using the GOLD-RTR receiver and interferometric waveforms using the PIR. Antennas $\mathbf{U}$ and $\mathbf{D}$ are mounted at the end of the boom.

The activity of measuring the height of the Antenna Reference Point (ARP) of these three antennas (U, N, D) above the sea will be executed as a number of independent but interrelated tasks: (1) Local surveying and monitoring of the antenna positions $(\mathbf{U}, \mathbf{N}, \mathbf{D})$ with respect to the RADAC altimeter (WG) and reference points (R1, R2) on the bridge. (2) RADAC WaveGuide free space radar altimeter measurements with $1 \mathrm{~cm}$ accuracy. (3) Connection of the bridge reference point (R2) to the national height datum (NAP), tide-gauge measurements from the RWS network, and optional GPS buoys measurements at nearby placement. (4) GPS buoys measurements (optional/backup).

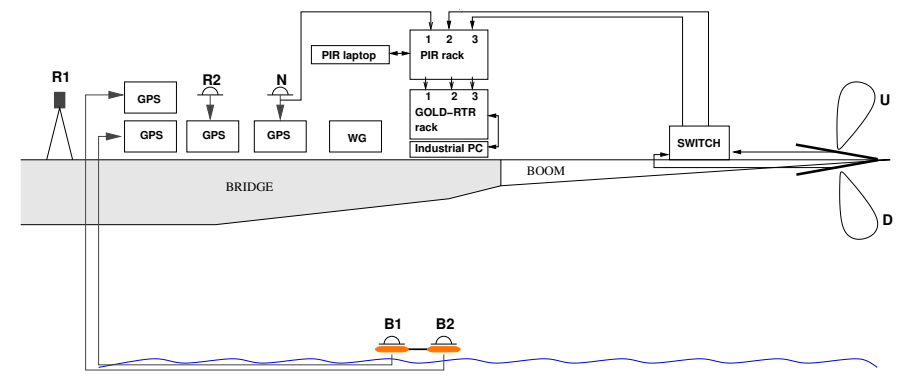

Fig. 6. Set-up for the bridge experiment.

\section{CONCLUSIONS}

A proof-of-concept instrument for PARIS-IOD, based on a full-custom dedicated GNSS-Reflections receiver has been developed, and the novel interferometric concept tested in several experiments using signals generated by a SPIRENT equipment. The preliminary analysis has resulted in the detection of 1-cm delay-jumps, using 33 group-delay observables of 1 second, with an associated dispersion of the order of 2-cm. A signal-to-noise degradation of the order of $\sim 3 \mathrm{~dB}$ with respect to the one expected for the Bridge Experiment would keep the 1-second sigma error below 3-cm.

A second experiment using real reflected signals obtained from a relatively high bridge $(\sim 15 \mathrm{~m})$ over estuary waters is planned for July 2010. The aim of the activity is to confirm the PARIS Interferometric Technique concept as an altimetric system able to provide $3-\mathrm{cm}$ resolution, as initially suggested by the presented preliminary results.

\section{REFERENCES}

[1] M. Martín-Neira, "A Passive Reflectometry and Interferometry System (PARIS): Application to Ocean Altimetry," ESA J., vol. 17, pp. 331-355, Dec. 1993.

[2] A. Rius, E. Cardellach, and M. Martin-Neira, "Altimetric analysis of the sea-surface GPS-reflected signals," IEEE Trans. Geosc. Remote Sensing, vol. 48, pp. 2119-2127, Apr. 2010.

[3] M. Martín-Neira, S. D’Addio, C. Buck, N. Floury, and R. Prieto-Cerdeira, "The Paris Ocean Altimeter In-orbit Demonstrator," Submitted to IEEE Trans. Geosc. Remote Sensing, June 2009.

[4] O. Nogués-Correig, E. Cardellach Galí, J. Sanz Campderròs, and A. Rius, "A GPS-Reflections Receiver That Computes Doppler/Delay Maps in Real Time," IEEE Trans. Geosc. Remote Sensing, vol. 45, no. 1, pp. 156174, Jan. 2007.

[5] SPIRENT Communications, "Simulating Multipath. Application Note DAN004-TM Issue 1-00," Tech. Rep., SPIRENT Communications.

[6] M. Martín-Neira, S. D’Addio, and C. Buck, "PARIS In Orbit Demonstrator: Part 3 - Geometry, Ionspheric Correction, Instrument Design, On-Board Calibration, Performance," in GNSS-R 08 Workshop. ESTEC, ESA, sep 2008. 